\title{
Rates and determinants of ventriculostomy-related infections during a hospital transition to use of antibiotic-coated external ventricular drains
}

\author{
Kelly Wright, Pharm.D., ${ }^{1}$ Polly Young, M.P.H., ${ }^{2}$ Cristina Brickman, M.D., ${ }^{3}$ \\ Teena Sam, Pharm.D., ${ }^{4}$ Neeraj Badjatia, M.D., ${ }^{2}$ Marcus Pereira, M.D., M.P.H., ${ }^{2}$ \\ E. Sander Connolly, M.D., 5 ANd Michael T. Yin, M.D., M.S. ${ }^{2}$ \\ ${ }^{1}$ Department of Pharmacy, Boston Medical Center, Boston, Massachusetts; ${ }^{2}$ Department of Medicine, \\ Columbia University, New York, New York; ${ }^{3}$ Department of Medicine, University of Pennsylvania, \\ Philadelphia, Pennsylvania; ${ }^{4}$ Department of Pharmacy, Yale-New Haven Hospital, New Haven, Connecticut; \\ and ${ }^{5}$ Department of Neurological Surgery, NewYork-Presbyterian Hospital/Columbia University, New York, \\ New York
}

\begin{abstract}
Object. The authors evaluated the rates of ventriculostomy-related infections (VRIs) after antibiotic-coated extraventricular drains (ac-EVDs) were introduced as the standard of care.

Methods. A retrospective chart review was conducted of adult patients admitted to NewYork-Presbyterian Hospital neurological intensive care unit in whom an EVD was placed between February 2007 and November 2009. excluding individuals receiving EVDs due to an infection of a primary device. Three time periods were defined depending on type of EVD in use: Period 1, conventional EVDs; Period 2, either ac-EVDs or conventional EVDs; and Period 3, ac-EVDs. Definite/probable VRIs that occurred during the 3 periods were evaluated and established as determinants of VRIs by using a Cox proportional hazards model. Prolonged systemic antibiotics were given for the duration of EVD placement in each of the 3 periods per institutional policy.

Results. Data from 141 individuals were evaluated; mean patient age was $53.8 \pm 17.2$ years and $54 \%$ were female. There were 2 definite and 19 probable VRIs. The incidence of definite/probable VRI (per 1000 person-catheter days) decreased from Period 1 to 3 (24.5, 16.2, and 4.4 in Periods 1, 2, and 3, respectively; $p<0.0001$ ). Patients with VRIs were more likely to be female than male $(23.7 \%$ vs $3.1 \%, p<0.003)$ and have had an EVD in place for a longer duration, although there was no significant difference among the 3 periods $(7.9 \pm 6.7$ [Period 1], $8.1 \pm 7.1$ [Period 2], and $8.6 \pm 5.8$ [Period 3] mean days; $p=0.87$, ANOVA). Analysis of effect modification in a stepwise model showed that period, age, and age and female interaction were significant predictors of VRIs. The period was the strongest predictor of VRI $(\mathrm{p}=0.0075)$. After adjustment for age and age and sex interaction, the survival rate was $53 \%$ at the end of Period 2 and $91 \%$ at the end of Period 3.

Conclusions. Rates of VRIs have decreased with the addition of ac-EVDs to the routine use of prolonged systemic antibiotics at the authors' institution.

(http://thejns.org/doi/abs/10.3171/2013.2.FOCUS12271)
\end{abstract}

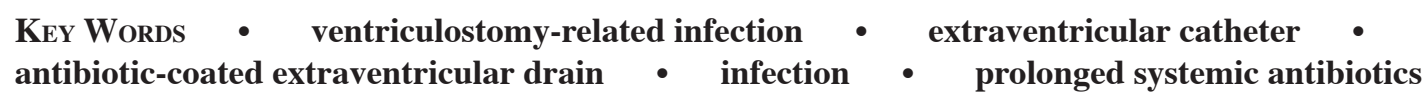

$\mathrm{E}$

xtraVentricular drains or EVDs provide a reliable means of monitoring and controlling intracranial pressure in patients with acute hydrocephalus secondary to subarachnoid, intracerebral, or intraventricular hemorrhages as well as from tumors that obstruct the CSF circulation. ${ }^{4}$ Their use, however, is associated with a higher risk of infection. Bacterial colonization of the cath-

Abbreviations used in this paper: ac-EVD = antibiotic-coated extraventricular drain; NICU = neurological intensive care unit; $\mathrm{PSA}=$ prolonged systemic antibiotics; $\mathrm{SAH}=$ subarachnoid hemor rhage; VRI = ventriculostomy-related infection. eters can lead to retrograde infection of the ventricles (also known as ventriculostomy-related infections or VRIs) followed by ventriculitis. The definitions for these infections vary. The Centers for Disease Control and Prevention defines adult ventriculitis by the presence of 1) a positive CSF culture or 2) signs of ventriculitis accompanied by use of antibiotics and evidence of CNS infection (at least one of the following: CSF pleocytosis; high CSF protein or low glucose level; organisms on CSF Gram stain; positive blood culture; positive antigen from CSF, blood, or urine; or positive serologies against pathogens associated with ventriculitis).${ }^{10}$ Because this definition does not re- 
quire positive CSF cultures and excludes the potential for catheter contaminants and colonization, many researchers instead accept the criteria proposed by Lozier et al., ${ }^{11}$ who define a VRI by the presence of 1 or more positive CSF cultures and/or Gram stains as well as abnormal CSF values (pleocytosis, high protein, low glucose).

The incidence of VRIs ranges from $2 \%$ to $27 \%$ depending on the study. ${ }^{4}$ Disparities have been ascribed to marked methodological differences, variability in definitions of infection, and sporadic use of antibiotics that often suppress detection of infection. A meta-analysis of 23 major studies comprising 5733 EVD insertions and 5261 patients demonstrated a cumulative rate of positive CSF cultures of $8.8 \%$ per patient or $8.1 \%$ per EVD. ${ }^{11}$

Several approaches have been advocated to prevent VRIs. One has been to use prophylactic antibiotics either at the time of EVD insertion, during the first 24 hours postinsertion, or in a prolonged systemic fashion (thus, PSA) while the EVD is in place. Evidence to support PSA is limited by the scarcity of adequately powered randomized controlled trials ${ }^{14,19}$ and conflicting results. ${ }^{5,11}$ As a result, there is no consistent practice regarding use of systemic antibiotics with EVDs and preference is largely based on tradition and training. ${ }^{12}$ A more recent approach is the use of commercially available ac-EVDs. In an industry-sponsored study, Zabramski et al..$^{20}$ randomized 288 patients to one of two groups: one receiving a VentriClear catheter, which is coated with rifampin and minocycline, and one receiving a conventional EVD. The ac-EVD group had a lower rate of CSF and catheter-tip culture positivity, but there was no comparison of outcomes based on a definition of VRI that included abnormal CSF parameters.

To reduce VRIs, NewYork-Presbyterian Hospital began using ac-EVDs containing rifampin and minocycline in late 2007. This study compares rates of VRIs in the NICU during 3 time periods: when only conventional EVDs were used (Period 1), when either conventional EVDs or ac-EVDs were used (Period 2), and when only ac-EVDs were used (Period 3). Prolonged systemic antibiotics, with cefazolin or vancomycin in the case of penicillin allergy, were used during all 3 time periods. The purpose of the investigation was to evaluate the rates of VRIs after ac-EVDs were introduced as the standard of care.

\section{Methods}

A retrospective chart review was conducted of adult patients admitted to the NICU who underwent placement of an EVD between February 1, 2007, and November 30, 2009. Eligible patients were 18 years and older and underwent placement of their first EVD after admission into the NICU. Individuals were excluded if they received an EVD for management of a prior EVD infection or if they had an EVD placed prior to the study period.

Subjects were divided into groups by type of EVD used depending on NICU admission date: Period 1 corresponded to a time when only conventional EVDs were used (February-September 2007), Period 2 to use of both conventional EVDs and ac-EVDs (October 2007-March
2008), and Period 3 to ac-EVDs only (April 2008-November 2008).

Catheters were inserted as separate procedures or in the setting of other surgical procedures such as craniotomies. Standard protocols were implemented for care of the ventricular catheter to avoid contamination of the systems. Per institutional policy, EVDs are usually inserted within 48 hours of admission to the NICU when indicated and are maintained at $10 \mathrm{~cm}$ above the external auditory meatus as long as the underlying reason for CSF diversion is present. The decision of whether to remove the EVD is based on the absence of raised intracranial pressure, the presence clinical symptoms, or the detection of radiographic signs referable to worsening hydrocephalus. Patients in whom these criteria are not met undergo ventriculoperitoneal shunting.

Electronic medical records were used to extract information on demographics; laboratory values; microbiology data from CSF, EVD catheter tips, tracheal aspirates, blood, and urine; use of antiinfective agents for prophylaxis and treatment of infection; EVD and other device (for example, spinal drains, ventricular peritoneal shunt, and so on) insertion and removal dates; indication for ventriculostomy catheter placement; and mortality.

The primary goal of the study was to compare the rates of definite and probable VRIs across the 3 periods. A VRI was defined as definite if there were clinical signs and symptoms of infection, abnormal CSF parameters, and a positive CSF culture; a VRI was defined as probable if clinical signs and symptoms and abnormal CSF parameters were present but the culture was negative; and no infection was defined as having no CSF abnormalities and a negative CSF culture. The secondary goal of the study was to evaluate predictors of VRIs.

\section{Statistical Analysis}

Baseline characteristics were compared between patients with and patients without VRIs by using chi-square or Fisher exact test for categorical variables and 2-sided ttests for normally distributed continuous variables. The incidence of VRI was calculated as cases per person-catheter days. We compared bivariate rates of VRI between EVD periods using the Kaplan-Meier survival curves. The logrank test was used to compare the differences in survival rates between changes in EVD type in the 3 observational periods. Effect modification was assessed by using interaction terms in a stepwise logistic regression. We used a criterion of $p<0.10$ for each variable or interaction term to be added to the model, and a $p<0.05$ criterion for the variable or interaction terms to be kept in the model after subsequent variables were added. These characteristics were further evaluated in a Cox proportional hazards model for risk estimates of VRI in patients with EVD placement during the 3 study periods. Time to infection was defined as days after EVD placement until diagnosis with a probable or definite VRI. Survival of infection was analyzed using a multivariate Cox regression model to estimate the simultaneous effects of independent variables on the incidence of VRIs. Proportional hazard ratios with 95\% confidence intervals were calculated. All analyses were conducted in SAS 9.2 (SAS Institute Inc.) 


\section{Rates of VRI after antibiotic-coated EVD introduction}

\section{Results}

\section{Patient Characteristics}

Between February 2007 and November 2009, 141 patients underwent placement of an EVD after admission to the NICU. Among patients receiving an EVD, 21 $(14.8 \%)$ of 141 developed a VRI during the course of their first EVD placement: 2 (9.5\%) of 21 were definite VRIs with a positive CSF culture (one with Escherichia coli and one with Staphylococcus epidermidis) and 19 (9.0\%) of 21 were probable VRIs with negative CSF cultures but with abnormal CSF values and clinical signs/symptoms. Table 1 shows the characteristics of these 2 groups. Patients who had a VRI were more likely to be female than male $(23.7 \%$ vs $3.1 \%, \mathrm{p}<0.003)$ and to have had the EVD in place for a longer duration (7.6 \pm 6.1 days vs $11.5 \pm 7.9$ days, $\mathrm{p}=0.04)$. The most common conditions that necessitated EVD placement were SAH (43.2\%) and intracranial hemorrhage (28.4\%), followed by tumors $(21.3 \%)$ and trauma $(2.8 \%)$.

\section{Rates of VRIs}

The proportion of VRIs among patients who required EVD insertion decreased from 23.5\% in Period 1 to $16.3 \%$ in Period 2 and $4.3 \%$ in Period 3 ( $\mathrm{p}=0.03$ ).
The incidence of definite/probable VRI (per 1000 personcatheter days) decreased significantly as well $(24.5,16.2$, and 4.4, respectively; $\mathrm{p}<0.001)$. Kaplan-Meier analysis (Fig. 1) indicated that incidence rates between periods were significantly different $(p=0.009, \log$-rank test).

Analysis of effect modification in a stepwise model showed that period, age, and age and female sex interaction were significant predictors of VRIs. Although duration of EVD therapy was significantly associated with VRIs, there was no significant difference seen between the 3 periods $(7.9 \pm 6.7$ mean days [Period 1], $8.1 \pm 7.1$ mean days [Period 2], $8.6 \pm 5.8$ mean days [Period 3]; $\mathrm{p}$ $=0.87$, ANOVA) and was therefore not evaluated in the model. Hazard ratios showed that period was the strongest predictor of VRI $(p=0.0075)$ (Table 2). After adjustment for age and age/sex interaction, the survival rate was 53\% at the end of Period 2 and $91 \%$ at the end of Period 3.

\section{Discussion}

We evaluated the effectiveness of ac-EVDs by comparing VRI rates in the NICU during 3 different treatment periods when we transitioned from using conventional EVDs to ac-EVDs. The proportion of EVD-treated patients with definite/probable VRIs decreased from $23.5 \%$

TABLE 1: Characteristics of study population by probable and definite VRI

\begin{tabular}{|c|c|c|c|}
\hline \multirow[b]{2}{*}{ Characteristic } & \multicolumn{2}{|c|}{ Value $(\%)$} & \multirow[b]{2}{*}{ p Value* } \\
\hline & No VRI $(n=120)$ & VRI $(n=21)$ & \\
\hline Period $\dagger$ & & & 0.0265 \\
\hline 1 & $39(76.5)$ & $12(23.5)$ & \\
\hline 2 & $36(83.7)$ & $7(16.3)$ & \\
\hline 3 & $45(95.7)$ & $2(4.3)$ & \\
\hline \multicolumn{4}{|l|}{ age (yrs) } \\
\hline mean \pm SD & $52.9 \pm 17.6$ & $58.7 \pm 14.7$ & 0.1733 \\
\hline range & $17-86$ & $18-78$ & \\
\hline \multicolumn{4}{|l|}{ sex } \\
\hline male & $62(96.9)$ & $3(3.1)$ & 0.0015 \\
\hline female & $58(76.3)$ & $18(23.7)$ & \\
\hline length of EVD insertion (days) & & & 0.0421 \\
\hline mean $\pm S D$ & $7.6 \pm 6.1$ & $11.5 \pm 7.9$ & \\
\hline range & $1-35$ & $4-40$ & \\
\hline EVD indication & & & 0.0331 \\
\hline $\mathrm{SAH}$ & $45(64.4)$ & $16(35.6)$ & \\
\hline $\mathrm{ICH}$ & $36(88.9)$ & $4(11.1)$ & \\
\hline tumor & $29(96.6)$ & $1(3.4)$ & \\
\hline trauma & $4(100)$ & $0(0)$ & \\
\hline hydrocephalus $\ddagger$ & $3(100)$ & $0(0)$ & \\
\hline infectious & $1(100)$ & $0(0)$ & \\
\hline other & $2(100)$ & $0(0)$ & \\
\hline
\end{tabular}

\footnotetext{
* The $p$ values are for differences in VRI status by chi-square or Fisher exact test for categorical variables and 2-sided t-test continuous variables.

$\dagger$ Conventional EVD catheters were used in Period 1 (2/07-9/07), ac-EVDs and conventional catheters were both used in Period 2 (10/07-03/08), and only ac-EVDs were used in Period 3 (04/08-11/08).

$\ddagger$ Cause of hydrocephalus not otherwise specified.
} 
K. Wright et al.

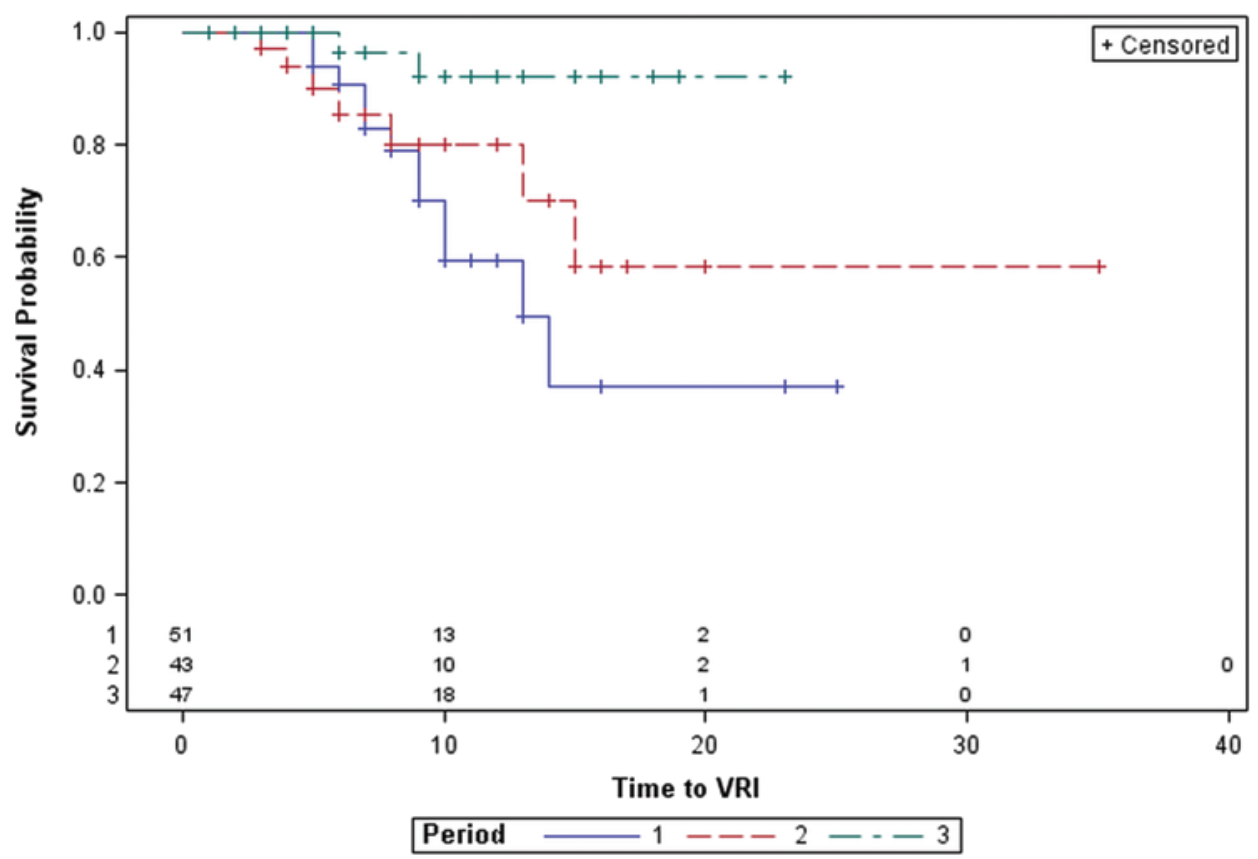

Fig. 1. Kaplan-Meier plot of VRI during 3 periods: Period 1, conventional EVD use; Period 2, mixed conventional and ac-EVD use; and Period 3, ac-EVD use.

in the period when only conventional EVDs were used, to $16 \%$ when conventional EVDs and ac-EVDs were both used, to $4.3 \%$ in the period when only ac-EVDs were used. This represents a risk ratio of 0.18 and an absolute risk reduction of $19.2 \%$ after transitioning from conventional to ac-EVD use. Female sex and duration of EVD insertion were significant predictors of VRIs in bivariate analyses; however, only sex and the interaction between sex and age were significant after logistic regression and analysis for effect modification. In an analysis of effect modification, time period was the strongest predictor of VRI.

The reduction in VRIs to $4.3 \%$ after introduction of ac-EVDs is similar to the rates seen in other retrospective and prospective studies of ac-EVDs use, where infection rates were decreased to $0.9 \%-5 \%$ per EVD. . $^{3,6-8,13,15,16,18}$ However, studies differ in terms of how they define a VRI and also by the presence of prolonged antibiotic prophylaxis, making direct comparisons with our study difficult. Zabramski et al., ${ }^{20}$ for example, reported results from a

TABLE 2: Multivariate-adjusted model of risk factors for probable and definite VRI after EVD placement

\begin{tabular}{llrl}
\hline \multicolumn{1}{c}{ Factor } & $\mathrm{HR}$ & \multicolumn{1}{c}{$95 \% \mathrm{Cl}$} & $\mathrm{p}$ Value \\
\hline Period & & & 0.0065 \\
2 & $0.471^{*}$ & $0.18-1.26$ & \\
3 & $0.091^{*}$ & $0.02-0.41$ & \\
sex & 0.045 & $0.001-1.43$ & 0.0790 \\
age & 1.051 & $1.01-1.09$ & 0.0140 \\
sex \& age interaction & 0.912 & $0.85-0.98$ & 0.0112 \\
\hline * Hazard ratios remained significant after adjustment with age and age/ \\
sex interaction.
\end{tabular}

6-center randomized clinical trial in which minocycline and rifampin ac-EVDs were compared with conventional EVDs, and almost all patients received PSA throughout the EVD placement period. Cerebrospinal fluid cultures from EVDs were obtained at regular intervals, and EVD catheter tips were cultured after removal. The ac-EVD group had a lower rate of CSF culture and catheter-tip culture positivity, but there was no comparison of outcomes based on a definition of VRI that included abnormal CSF parameters, making it difficult to discern whether the results reflect a difference in rates of VRI or just catheter colonization. Other studies have also used a positive CSF culture to define VRI, but they vary in their sampling frame, from systematic CSF surveillance ${ }^{2,7,8,18}$ to sampling only when there are clinical indications such as fever or leukocytosis., $3,15,16$ Among published observational studies, the largest was conducted by Harrop et al., ${ }^{8}$ in which 1961 ventriculostomies were observed over 6 years during 5 different periods of VRI control. Rates of VRI were lower in both periods during which ac-EVDs were used $(0.9 \%-1.0 \%)$ than in periods when a conventional EVD was used (6.7\%-8.2\%). A VRI was defined strictly as having 2 positive CSF cultures obtained from the EVD and a concurrent increase in CSF white blood cell counts. Prolonged systemic antibiotics were not used during any study period. Use of antibiotics varied in other studies, ranging from use of periprocedural antibiotics, ${ }^{1,9,16}$ to partial use of PSA, ${ }^{3}$ to consistent use of PSA. ${ }^{18}$ Despite the difficulty in combining data with such differences in outcome definition and PSA practice, a recent meta-analysis by Sonabend and coworkers ${ }^{17}$ pooled 1 randomized control trial and 3 observational studies to estimate a risk ratio of 0.19 (95\% CI $0.07-0.52)$ for ac-EVD use in the prevention of VRIs, which was very similar to our study findings. 
The predominant indication for EVD placement in our study was SAH (43.3\%), similar to that at other institutions. ${ }^{1,16,20}$ Duration of ac-EVD therapy was significantly higher in the group with VRIs $(11.5 \pm 7.9$ vs 7.6 $\pm 6.1, \mathrm{p}=0.04)$ and is a commonly observed risk factor for VRIs. ${ }^{3,9,11}$ In our study, females were 6 times more likely to have VRIs after EVD insertion than males (OR $6.4, \mathrm{p}<0.005)$. This could be because women are much more likely than men to have SAH, and VRIs were more likely to occur in patients with SAH than other conditions requiring EVD placement. In a study by Sloffer et al., ${ }^{16}$ SAH accounted for $71.7 \%$ of indications for EVD and the authors' analytical cohort also had a significantly higher proportion of women (61 women vs 39 men). Another study that evaluated VRI risk factors ${ }^{3}$ in a predominantly male cohort (79\%) also found that males were less likely than females to develop VRI (9\% vs 33\%, p = 0.009). Additional prospective and randomized studies are necessary to discern whether the observed increased risk of VRI in women is due to confounding factors or is indicative of biological susceptibility.

In our institution, PSA with cefazolin or vancomycin for penicillin-allergic patients was given to all patients from time of insertion to removal of the EVD. While there is some data suggesting that PSA prevents VRIs seen with conventional EVDs, $3,14,17$ there are no data to suggest that PSA provides additional clinical or microbiological benefit in combination with ac-EVDs. There are several risks associated with prolonged antibiotic exposure including development of antibiotic resistance, adverse effects of the antibiotics such as antibiotic-associated diarrhea, and Clostridium difficile infection. We did not observe an increase in the number of resistant organisms in the available sputum or urine cultures of patients receiving cefazolin or vancomycin for their EVD; however, cultures were sent only when clinically indicated. To truly assess the impact of PSA with EVDs, participants' samples would have to be systematically cultured at regular intervals.

The 2 main limitations of this study are its retrospective design and its limited sample size. Definitions for infection were highly reliant upon documentation in the medical record and opinions of the primary team. Variations in documentation and practice deviations among providers cannot be accounted for in the present study; however there is nothing to suggest that these were significantly different in any of the 3 time periods. The modest sample size limited our ability to detect smaller differences in infection rates or other risk factors.

\section{Conclusions}

In this retrospective cohort review, we observed a decrease in rates of VRIs during a transition from use of conventional EVD catheters to use of ac-EVDs. Even though PSA was used throughout the 3 periods, VRI rates were significantly reduced with the introduction of ac-EVDs. These results suggest that routine use of PSA could be discontinued with utilization of ac-EVDs, thereby limiting the potential adverse effects and development of resistant colonizing organisms associated with antibiotic exposure.

\section{Disclosure}

This publication was supported by the National Center for Research Resources and the National Center for Advancing Translational Sciences, NIH, through Grant No. UL1 RR024156. The content is solely the responsibility of the authors and does not necessarily represent the official views of the NIH.

Author contributions to the study and manuscript preparation include the following. Conception and design: Wright, Young, Brickman, Sam, Pereira, Yin. Acquisition of data: Yin. Analysis and interpretation of data: Wright, Young, Brickman, Badjatia, Yin. Drafting the article: Wright, Young, Brickman, Badjatia, Yin. Critically revising the article: Wright, Young, Brickman, Badjatia, Pereira, Yin. Reviewed submitted version of manuscript: all authors. Approved the final version of the manuscript on behalf of all authors: Wright. Statistical analysis: Young, Badjatia, Yin. Study supervision: Badjatia, Connolly, Yin.

\section{References}

1. Abla AA, Zabramski JM, Jahnke HK, Fusco D, Nakaji P: Comparison of two antibiotic-impregnated ventricular catheters: a prospective sequential series trial. Neurosurgery 68 : 437-442, 2011

2. Alleyne CH Jr, Hassan M, Zabramski JM: The efficacy and cost of prophylactic and perioprocedural antibiotics in patients with external ventricular drains. Neurosurgery 47: 1124-1129, 2000

3. Arabi Y, Memish ZA, Balkhy HH, Francis C, Ferayan A, Al Shimemeri A, et al: Ventriculostomy-associated infections: incidence and risk factors. Am J Infect Control 33:137-143, 2005

4. Beer R, Lackner P, Pfausler B, Schmutzhard E: Nosocomial ventriculitis and meningitis in neurocritical care patients. J Neurol 255:1617-1624, 2008

5. Dasic D, Hanna SJ, Bojanic S, Kerr RSC: External ventricular drain infection: the effect of a strict protocol on infection rates and a review of the literature. Br J Neurosurg 20:296-300, 2006

6. Demetriades AK, Bassi S: Antibiotic resistant infections with antibiotic-impregnated Bactiseal catheters for ventriculoperitoneal shunts. Br J Neurosurg 25:671-673, 2011

7. Eymann R, Chehab S, Strowitzki M, Steudel WI, Kiefer M: Clinical and economic consequences of antibiotic-impregnated cerebrospinal fluid shunt catheters. J Neurosurg Pediatr 1:444-450, 2008

8. Harrop JS, Sharan AD, Ratliff J, Prasad S, Jabbour P, Evans $\mathrm{JJ}$, et al: Impact of a standardized protocol and antibioticimpregnated catheters on ventriculostomy infection rates in cerebrovascular patients. Neurosurgery 67:187-191, 2010

9. Hoefnagel D, Dammers R, Ter Laak-Poort MP, Avezaat CJJ: Risk factors for infections related to external ventricular drainage. Acta Neurochir (Wien) 150:209-214, 2008

10. Horan TC, Andrus M, Dudeck MA: CDC/NHSN surveillance definition of health care-associated infection and criteria for specific types of infections in the acute care setting. Am J Infect Control 36:309-332, 2008 (Erratum in Am J Infect Control 36:655, 2008)

11. Lozier AP, Sciacca RR, Romagnoli MF, Connolly ES Jr: Ventriculostomy-related infections: a critical review of the literature. Neurosurgery 51:170-182, 2002

12. McCarthy PJ, Patil S, Conrad SA, Scott LK: International and specialty trends in the use of prophylactic antibiotics to prevent infectious complications after insertion of external ventricular drainage devices. Neurocrit Care 12:220-224, 2010

13. Parker SL, Attenello FJ, Sciubba DM, Garces-Ambrossi GL, Ahn E, Weingart J, et al: Comparison of shunt infection incidence in high-risk subgroups receiving antibiotic-impregnated versus standard shunts. Childs Nerv Syst:77-83, 2009 


\section{K. Wright et al.}

14. Poon WS, Ng S, Wai S: CSF antibiotic prophylaxis for neurosurgical patients with ventriculostomy: a randomised study. Acta Neurochir Suppl 71:146-148, 1998

15. Rivero-Garvía M, Márquez-Rivas J, Jiménez-Mejías ME, Neth $\mathrm{O}$, Rueda-Torres AB: Reduction in external ventricular drain infection rate. Impact of a minimal handling protocol and antibiotic-impregnated catheters. Acta Neurochir (Wien) 153: 647-651, 2011

16. Sloffer CA, Augspurger L, Wagenbach A, Lanzino G: Antimicrobial-impregnated external ventricular catheters: does the very low infection rate observed in clinical trials apply to daily clinical practice? Neurosurgery 56:1041-1044, 2005

17. Sonabend AM, Korenfeld Y, Crisman C, Badjatia N, Mayer SA, Connolly ES Jr: Prevention of ventriculostomy-related infections with prophylactic antibiotics and antibiotic-coated external ventricular drains: a systematic review. Neurosurgery 68:996-1005, 2011

18. Tamburrini G, Massimi L, Caldarelli M, Di Rocco C: Antibiotic impregnated external ventricular drainage and third ventriculostomy in the management of hydrocephalus associated with posterior cranial fossa tumours. Acta Neurochir (Wien) 150:1049-1056, 2008
19. Wyler AR, Kelly WA: Use of antibiotics with external ventriculostomies. J Neurosurg 37:185-187, 1972

20. Zabramski JM, Whiting D, Darouiche RO, Horner TG, Olson $\mathrm{J}$, Robertson C, et al: Efficacy of antimicrobial-impregnated external ventricular drain catheters: a prospective, randomized, controlled trial. J Neurosurg 98:725-730, 2003

Manuscript submitted August 1, 2012.

Accepted February 20, 2013.

Preliminary data were presented in poster form at the 48th Annual Meeting of the Infectious Diseases Society of America, Vancouver, British Columbia, Canada, October 21-24, 2010.

Please include this information when citing this paper: DOI: 10.3171/2013.2.FOCUS12271.

Address correspondence to: Kelly Wright, Pharm.D., Department of Pharmacy, Boston Medical Center, 1 Boston Medical Center Place, Boston, Massachusetts 02118. email: Kelly.Wright @bmc.org. 